\title{
Flow control in s-shaped air intake diffuser of gas turbine using proposed energy promoters
}

\author{
Raed A. Jessam ${ }^{1,2}$, Hussain H. Al-Kayiem ${ }^{1,}{ }^{*}$, Mohammed S. Nasif ${ }^{1}$ \\ ${ }^{1}$ Mech. Eng. Dept., Universiti Teknologi PETRONAS, 32610 Bandar Seri Iskandar, Malaysia \\ ${ }^{2}$ Electromechanical Engineering Department, University of Technology, Baghdad, Iraq
}

\begin{abstract}
This paper presents an experimental and numerical investigation of the flow control in an air intake S-shaped diffuser with and without energy promoters. The S-shaped diffuser had an area ratio 3.1 and turning angle of $45^{\circ} / 45^{\circ}$. The proposed energy promoter was named as stream line sheet energy promoter. Computational Fluid Dynamics simulation was performed through commercial ANSYS-FLUENT 16.2 software. The measurements were made inside annular subsection, $45^{\circ}$ from $360^{\circ}$ of the complete annular shape of the diffuser, at Reynolds number $5.8 \times 10^{4}$ and turbulence intensity $4.1 \%$. Results for the bare S-shaped diffuser (without energy promoters) showed the flow structures within the S-shaped diffuser were dominated by counter-rotating vortices and boundary layer separation especially in the outer surface. The combination of the adverse pressure gradient at the first bend of outer surface and upstream low momentum wakes caused the boundary layer to separate early. The combinations of proposed energy promoters were installed on the inner and outer surfaces at three installation planes. The use of energy promoters resulting in significantly decreased the outer surface boundary layer separation with consequential improving the static pressure coefficient and reduction of total pressure losses.
\end{abstract}

\section{Introduction}

Air intakes are main component of breathing aero-engines. The main task is to sustain high pressure recovery and reduce the inflow velocity to avoid presence of shock waves inside the diffuser part of the air intake. This part is strongly affecting the engine performance. Among the most commonly applied types of intakes is the S-shaped diffuser, which guides air from around a plane body to the engine inlet [1]. The S-shaped diffusers have centerline curvatures and cross-sectional areas that increase along the flow direction. Owing to this centerline curvature, cross-stream pressure gradients build up and increase secondary flows. This phenomenon creates cross-flow vortices within the boundary layer that generate a non-uniform pressure profile at the gas turbine engine face. This profile is called the aerodynamic inlet plane AIP. In addition, the adverse streamwise pressure gradient caused by the increasing cross-sectional area can also lead to flow separation, which is consider the major problem of S-shaped diffuser.

- Corresponding author: hussain_kayiem@utp.edu.my 
To achieve acceptable performance, an S-shaped diffuser must incur minimal total pressure losses and facilitate almost uniform flow with small cross-flow velocity components at the AIP [2].

Many techniques have been used to improve the performance of S-shaped diffuser. One of the important technique is the passive flow control, vortex generators (VGs), which modifies the boundary layer fluid motion by bringing momentum from the outer flow region into the inner flow region of the wall bounded flow. Use of a set of VGs located on the outer and inner surfaces has been proposed for restructures the flow field [3]. Ref. [4] have been conducted experiments to study the effect of fences and VGs in terms of reducing exit flow distortion and improving total pressure recovery in two-dimensional, rectangular S-duct diffusers with different radius ratios. Ref. [5] investigated various low-profile VGs, counterrotating vanes, single vane, forwards wedge, and backwards wedge referred to as sub boundary-layer vortex generators.

In the same direction, [6] investigated experimentally another type of VG named as LowProfile Vortex Generators (LPVGs) to reduce the total pressure distortion in S-shaped diffuser. A low-profile wishbone type VG was used to improve the total pressure distortions and recovery performance of a diffusing S-duct by [7]. Ref. [8] used LPVGs within the first bend of this S-shaped intermediate turbine diffuser to study the influence of passive flow control devices on to separation. [9], have tested experimentally counter-rotating and corotating VGs configurations, installed on the casing of S-duct, on the flow development within an aggressive interturbine duct.

The objective of the present study is to investigate the contribution of different configurations of stream line EPs on the flow control in S-shaped diffuser by experiments through a test rig was set up for this purpose and numerically by three dimensional (3D) simulations by commercial ANSYS-FLUENT 16.2 software. Three proposed configurations of EPs have been investigated which have different numbers of EPs at three installation planes on the top and bottom surfaces of S-shaped diffuser. Total pressure loss coefficient, $C_{T L}$, static pressure recovery coefficient, $C_{P R}$, have been adopted as indicators to highlight the contribution of the EPs on controlling the flow and enhance the diffuser performance.

\section{Experimental methodology}

\subsection{S-shaped Diffuser Model}

The geometry of the S-shaped diffuser used in this study is shown in Figure 1. It was designed based on area ratio AR of 3.1. The radius of curvature, $R C=280 \mathrm{~mm}$, the turning angle, $\beta=45^{\circ}$. The total length of the diffuser, $L=526 \mathrm{~mm}$, and the hydraulic diameter, $D_{h}=$ $65 \mathrm{~mm}$. The modeled diffuser has a constant area conduit at the inlet with $65 \mathrm{~mm}$ long x 65 $\mathrm{mm}$ height, for smooth air inflow, and constant area conduit at the outlet with $65 \mathrm{~mm}$ long $\mathrm{x} 125 \mathrm{~mm}$ height. The selected geometry of the model is representing $1 / 8$ segment of the entire annulus. I.e., it is only $45^{\circ}$ section of the $360^{\circ}$ of the complete circular shape of the diffuser; is going to be named as annular subsection. Most if not all the previously reported studies, the investigator selected similar cross section, but with straight inner and outer surfaces. In the present work, the inner and outer surfaces have been simulated as curved inner and outer surfaces, which represent the actual case of the diffuser segment. 


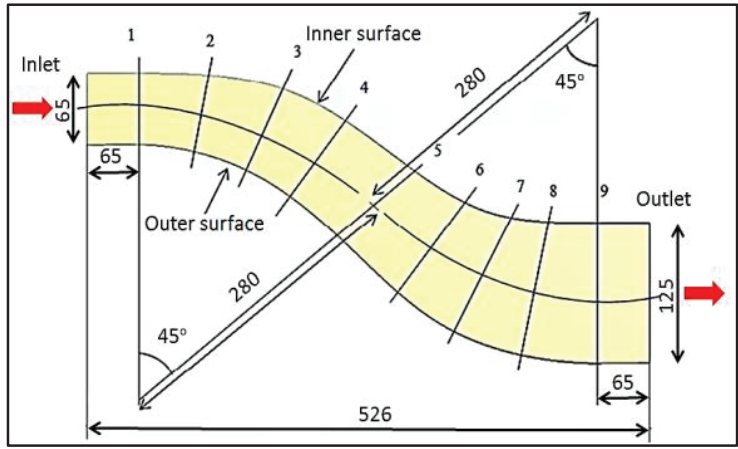

Fig. 1. Geometry of the modeled S-shaped diffuser and plane of interest (all dimensions in $\mathrm{mm}$ )

KIMO Multifunction AMI300 was connected with hot wire for measuring the velocity at these planes, also it has been used for measuring the turbulence intensity experimentally at the inlet plane. Also, it related to Pitot static tube for pressure measurements at 10 planes rated for $\pm 0.2 \mathrm{pa}$. At each plane, measurements are taken over a period of $10 \mathrm{~s}$ which allows for the calculation of a mean value along with its standard deviation. The main parts of test rig are shown in Figure 2. The inlet velocity of $14.4 \mathrm{~m} / \mathrm{s}$ and the Reynolds numbers of $5.8 \times 10^{4}$ obtained depending on the inlet hydraulic diameter of S-shaped diffuser. Also, the measured turbulence intensity by experiment at the inlet plane of S-shaped diffuser was $4.1 \%$. These inlet velocity and inlet turbulence intensity have been used an inlet boundary conditions for CFD simulation.

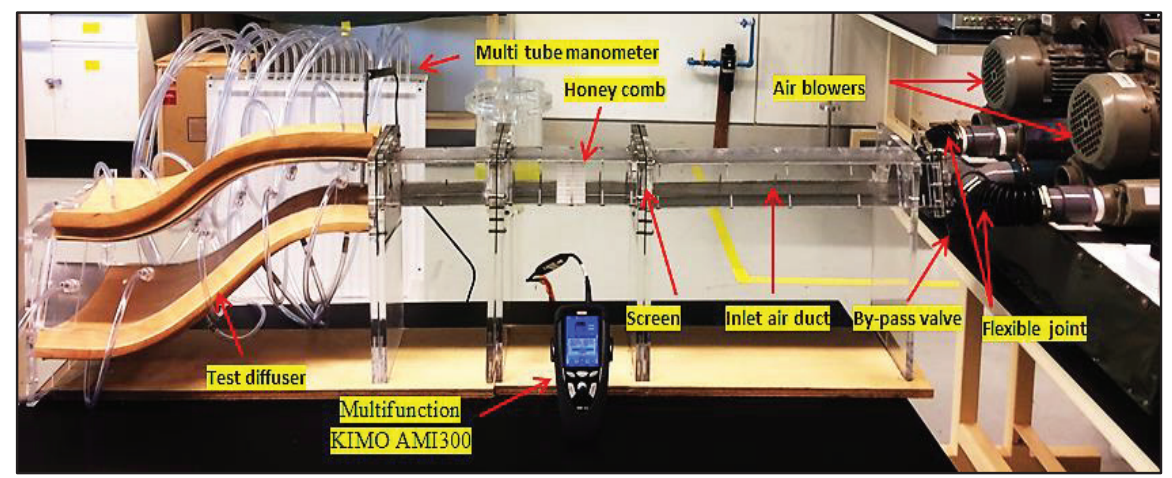

Fig. 2. S-shaped diffuser Test Apparatus

\subsection{Proposed EPs Configurations}

Three configurations of stream line sheet EPs were employed for elimination the flow separation region and delay separation starting. Also, to improve the flow pattern at the exit plane of the S-shaped diffuser by increase the static pressure recovery and reduce the total pressure loss. Figure 3 shows the investigated installation planes, while figure 4 shows the proposed EP geometry with the geometrical specifications. The EPs have been distributed on the inner and outer surfaces at plane 3,4 and 5 as bellow:

- At plane 3: 5 EPs on the inner surface and 7 EPs on the outer surface.

- At plane 4: 5 EPs on the inner surface and 7 EPs on the outer surface.

- At plane 5: 6 EPs on the inner surface and 8 EPs on the outer surface. 

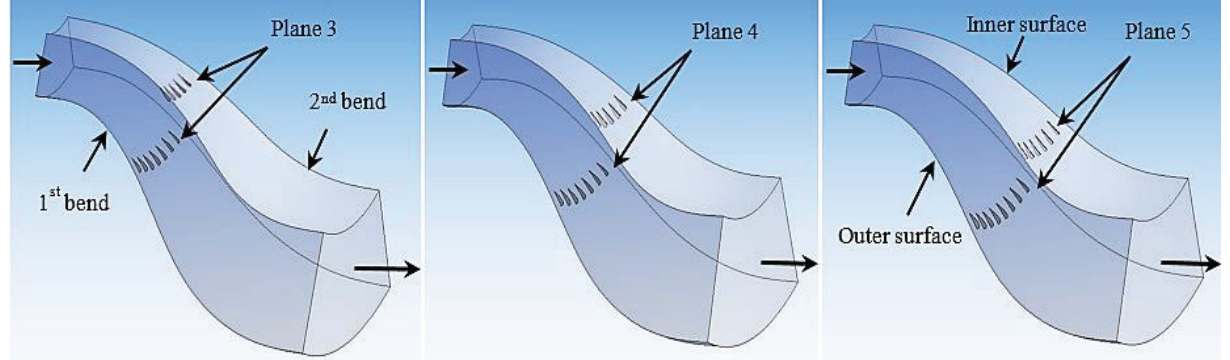

Fig. 3. Geometry of Co-rotating stream line sheet EP

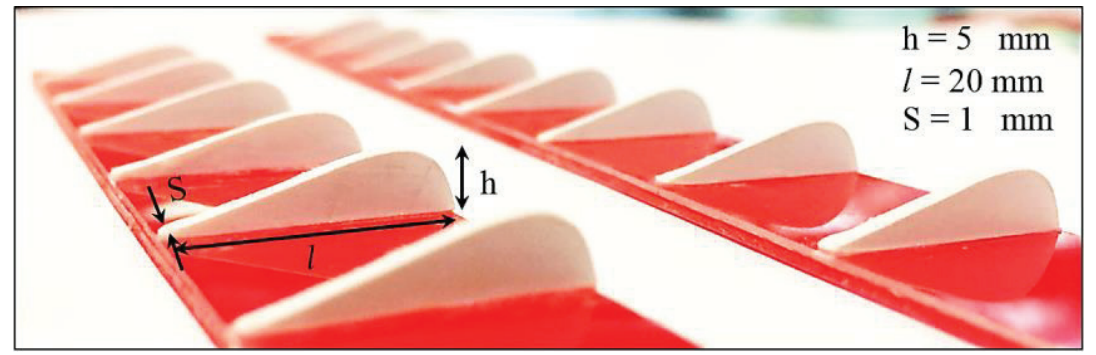

Fig. 4. Co-rotating stream line sheet EPs on the inner and outer surfaces of S-shaped diffuser at plane 3,4 and 5 .

\section{Numerical methodology}

This section provides information on the S-shaped diffuser grid generation, the governing equations, boundary conditions, mesh independency checking. Also, the validation of the turbulence models results with the experimental data has been presented.

\subsection{Governing equations and boundary conditions}

The governing equations for continuity and momentum, three-dimensional, steady state, Reynolds Average Navier Stokes (RANS) equations were numerically solved turbulent incompressible flows of air:

Continuity equation in coordinates $(\mathrm{x}, \mathrm{y}, \mathrm{z})$

$$
\frac{\partial u}{\partial x}+\frac{\partial v}{\partial y}+\frac{\partial w}{\partial z}=0
$$

$\mathrm{X}$ - Momentum equation:

$u \frac{\partial u}{\partial x}+v \frac{\partial u}{\partial y}+w \frac{\partial u}{\partial z}=-\frac{1}{\rho} \frac{\partial P}{\partial x}+v\left[\frac{\partial^{2} u}{\partial x^{2}}+\frac{\partial^{2} u}{\partial y^{2}}+\frac{\partial^{2} u}{\partial z^{2}}\right]+\frac{1}{\rho}\left[\frac{\partial\left(-\rho \overline{u^{\prime 2}}\right)}{\partial x}+\frac{\partial\left(-\rho \overline{u^{\prime} v^{\prime}}\right)}{\partial y}+\frac{\partial\left(-\rho \overline{u^{\prime} w^{\prime}}\right)}{\partial z}\right]$

$\mathrm{Y}$ - Momentum equation:

$u \frac{\partial v}{\partial x}+v \frac{\partial v}{\partial y}+w \frac{\partial v}{\partial z}=-\frac{1}{\rho} \frac{\partial P}{\partial y}+v\left[\frac{\partial^{2} v}{\partial x^{2}}+\frac{\partial^{2} v}{\partial y^{2}}+\frac{\partial^{2} v}{\partial z^{2}}\right]+\frac{1}{\rho}\left[\frac{\partial\left(-\rho \overline{u^{\prime} v^{\prime}}\right)}{\partial x}+\frac{\partial\left(-\rho \overline{v^{\prime 2}}\right)}{\partial y}+\frac{\partial\left(-\rho \overline{v^{\prime} w^{\prime}}\right)}{\partial z}\right]$

$\mathrm{Z}$ - Momentum equation:

$u \frac{\partial w}{\partial x}+v \frac{\partial w}{\partial y}+w \frac{\partial w}{\partial z}=-\frac{1}{\rho} \frac{\partial P}{\partial x}+v\left[\frac{\partial^{2} w}{\partial x^{2}}+\frac{\partial^{2} w}{\partial y^{2}}+\frac{\partial^{2} w}{\partial z^{2}}\right]+\frac{1}{\rho}\left[\frac{\partial\left(-\rho \overline{u^{\prime} w^{\prime}}\right)}{\partial x}+\frac{\partial\left(-\rho \overline{v^{\prime} w^{\prime}}\right)}{\partial y}+\right.$

$\left.\frac{\partial\left(-\rho \overline{w^{\prime 2}}\right)}{\partial z}\right]$ 
The flow field was predicted by solving the set of equations from (1) to (4). The 3D RNG $k-\varepsilon$ model for simulating the turbulence quantities has been adopted. The enhanced wall treatment was selected to overcome with high complex three dimensional near wall flow phenomena of diffusers. In the present study, the first grid point of $y+\approx 1.4$ and the number cells around 25 cell within the inner layer of wall. The flow field was predicted by solving the set of equations from (1) to (4). The boundary conditions were used for simulations as summarized in Table1.

Table 1. Description of boundary conditions

\begin{tabular}{|c|c|c|c|c|c|c|c|}
\hline \multicolumn{2}{|c|}{$\begin{array}{c}\text { Inlet boundary } \\
\text { conditions }\end{array}$} & \multicolumn{2}{c|}{$\begin{array}{c}\text { Working fluid } \\
\text { properties (air) }\end{array}$} & \multicolumn{2}{c|}{$\begin{array}{c}\text { Outlet boundary } \\
\text { conditions }\end{array}$} & \multicolumn{2}{c|}{$\begin{array}{c}\text { Wall boundary } \\
\text { conditions }\end{array}$} \\
\hline $\begin{array}{c}\text { Velocity } \\
\text { inlet }(\mathrm{m} / \mathrm{s})\end{array}$ & 14.4 & $\begin{array}{c}\text { Density } \\
\left(\mathrm{kg} / \mathrm{m}^{3}\right)\end{array}$ & 1.1649 & $\begin{array}{c}\text { Pressure } \\
\text { specified (Pa) }\end{array}$ & 0 & $\begin{array}{c}\text { Share } \\
\text { condition }\end{array}$ & $\begin{array}{c}\text { No } \\
\text { slip }\end{array}$ \\
\hline $\begin{array}{c}\text { Turbulence } \\
\text { intensity }\end{array}$ & $4.1 \%$ & $\begin{array}{c}\text { Viscosity } \\
(\mathrm{kg} / \mathrm{m} . \mathrm{s})\end{array}$ & $1.85 \times 10^{-5}$ & & & & \\
\hline
\end{tabular}

\subsection{Grid generation}

The S-shaped diffuser used in the experiments has been modeled by modular design ANSYS 16.2, a grid generation by ANSYS - FLUENT 16.2. Figure 5 shows the structured mesh along the bare S-shaped diffuser (without EPs) and unstructured mesh of S-shaped diffuser with EPs. The S-shaped diffuser used in the experiments has been modeled by modular design ANSYS 16.2, a grid generation by ANSYS - FLUENT 16.2. Consistently with the experimental setup, a $65 \mathrm{~mm}$ entrance length (upstream the S-shaped diffuser) and a $65 \mathrm{~mm}$ long extension (downstream the S-shaped diffuser) are used in the numerical model.

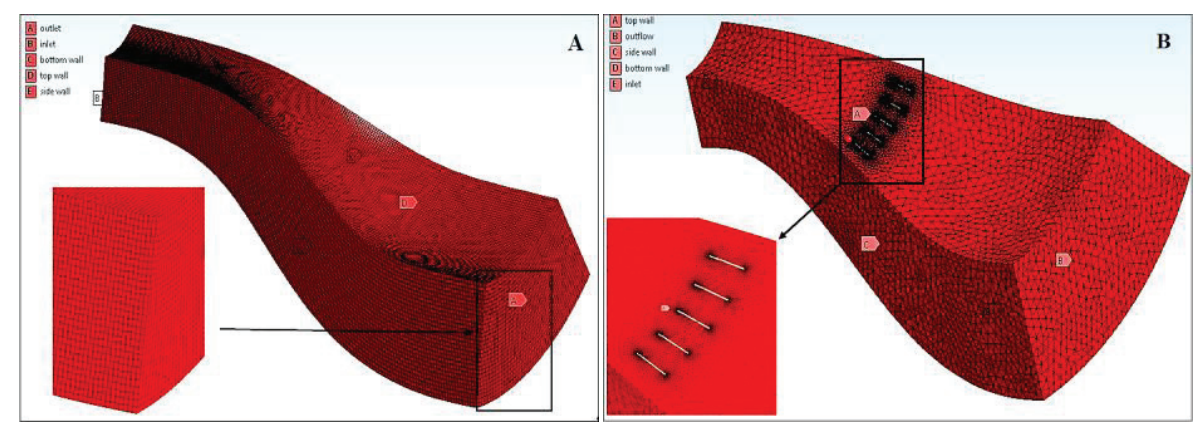

Fig. 5. Generated mesh of S-shaped diffuser: A. structured mesh without EPs, B. unstructured mesh with EPs.

\subsection{Performance parameters}

The main important parameters are using for evaluation the performance of S-shaped diffuser in the current study are:

\section{Static Pressure Recovery Coefficient $C_{P R}$}

It represents the magnitude by which kinetic energy has been converted into pressure energy due to diffusing action at any location along the S-shaped diffuser, it is represented by the equation: 


$$
C_{P R}=\frac{P_{S}-P_{S i}}{\frac{1}{2} \rho U_{a v i}^{2}}
$$

Where: $P_{s} \quad$ static pressure $(\mathrm{Pa})$

$P_{s i} \quad$ static pressure at the inlet plane $(\mathrm{Pa})$

$U_{a v i}$ average inlet velocity $(\mathrm{m} / \mathrm{s})$

\section{Total Pressure Loss Coefficient $C_{T L}$}

It is a measure of how much total pressure is lost as a proportion of the mean inlet dynamic pressure due to viscous forces and turbulent mixing, this defined by the equation

$$
C_{T L}=\frac{P_{t i}-P_{t}}{\frac{1}{2} \rho U_{a v i}^{2}}
$$

where: $P_{t i}$ inlet total pressure $(\mathrm{Pa})$

$P_{t} \quad$ total pressure $(\mathrm{Pa})$

\section{Results and discussion}

This section will be explaining the experimental and numerical results through the performance parameters $C_{P R}$ and $C_{T L}$. The study cases were named as in Table2:

Table 2. Description of experimental and numerical study cases of S-shaped diffuser

\begin{tabular}{|l|l|l|l|}
\hline \multicolumn{2}{|c|}{ Experimental Study } & \multicolumn{2}{c|}{ Numerical Study by CFD } \\
\hline Name & Description & Name & Description \\
\hline Exp.1 & Bare S-shaped diffuser & CFD1 & Bare S-shaped diffuser \\
\hline Exp.2 & EPs installed at plane 3 & CFD2 & EPs installed at plane 3 \\
\hline Exp.3 & EPs installed at plane 4 & CFD3 & EPs installed at plane 4 \\
\hline Exp.4 & EPs installed at plane 5 & CFD4 & EPs installed at plane 5 \\
\hline
\end{tabular}

Figure 6-A shows the variation of static pressure recovery coefficient $C_{P R}$ along the length of the S-shaped diffuser. The $C_{P R}$ values that were computed experimentally by test rig measurements have been compared with those were computed numerically by CFD. The $C_{P R}$ values, for CFD1, was increased in the initial phase up to plane 3 around $X / L \approx 0.35$, after that it decrease due to flow separation until flow reattachment occurs somewhere before plane 7 at $X / L \approx 0.68$ and then it increases steadily till the exit plane. For the experimental measurements, the trend of $C_{P R}$ similar to the $C_{P R}$ by CFD with some deviations could be observed in the location and length of the separated flow region. $C_{P R}$ curve increase in the initial phase up to around $X / L \approx 0.3$ and then it decreases due to flow separation. The flow separation occurs early with experimental investigation, also flow reattachment occurs after plane 7. While, the values and the trend of $C_{P R}$ were improved for both the experimental and numerical investigations with applying EPs on the inner and outer surfaces at the three installation planes of diffuser. Generally, the applying EPs at planes 3, 4 and 5 was improved the experimental and numerical values of $C_{P R}$ of $28 \%$ and $30 \%$ respectively. The Exp. 4 and CFD4 cases, give the best improvement of $C_{P R}$ among the other cases, this improvement resulting from decrease the flow velocity and flow separation region especially on the outer surface as shown in Figure 6-B. The EPs were modified the boundary layer fluid flow by bringing momentum from the outer flow region into the inner flow region of the wall. 


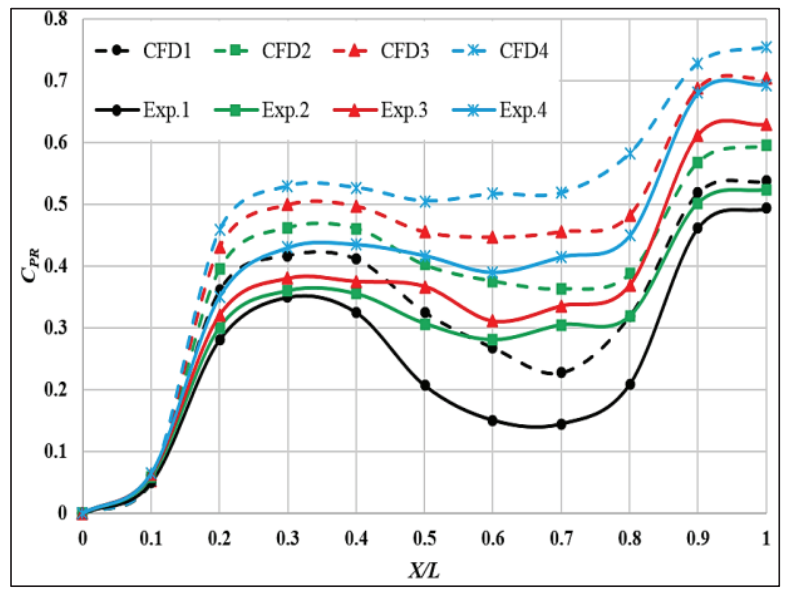

A

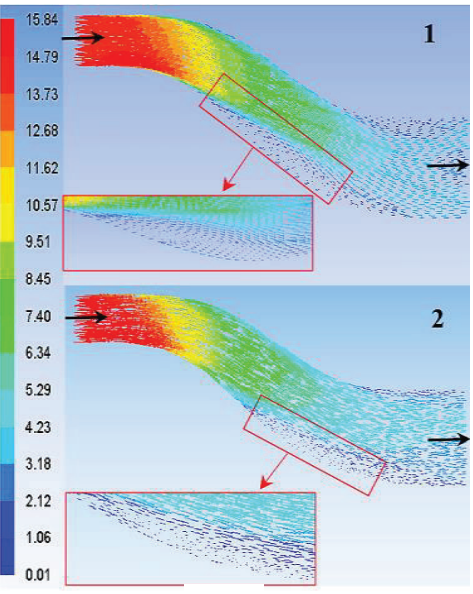

B

Fig. 6. (A)Variation of $C_{P R}$ along the length of S-shaped diffuser, (B) Velocity vectors with separation regions of S-shaped diffuser: 1.Bare (without EPs), 2. With EPs

Figure 7-A shows that the $C_{T L}$ decreases with the applying EPs on the inner and outer surfaces at planes 3, 4 and 5 as compared with bare S-shaped diffuser. Applying the EPs at plane 5 gives the best improvement in $C_{T L}$ for CFD and experimental results of $18 \%$ and $11 \%$ as compared with bare S-shaped diffuser, respectively. The reduction in $C_{T L}$ resulting from decrease of total pressure along the diffuser and separation region especially on the outer surface, as shown in Figure 7-B.

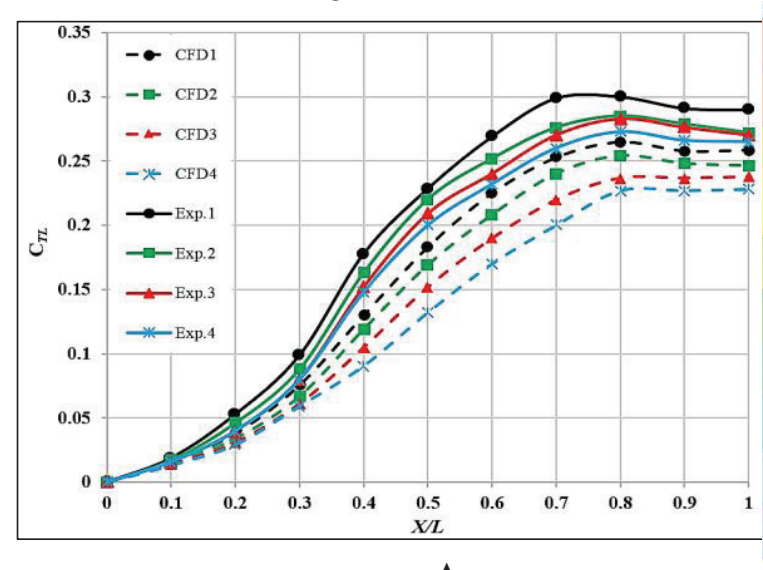

A

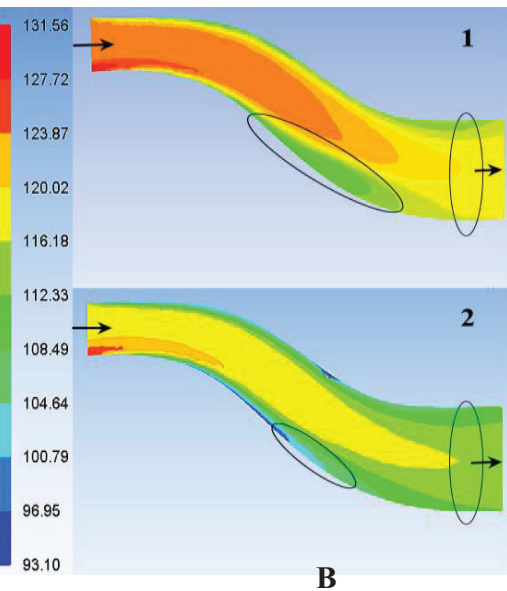

B

Fig. 7. (A) Values of $C_{T L}$ along the length of bare S-shaped diffuser and S-shaped diffuser with EPs, B. Total pressure contours of S-shaped diffuser1.Bare (without EPs), 2. With EPs.

\section{Conclusions}

Experimental and numerical studies have been conducted on bare S-shaped diffuser (without EPs) and S-shaped diffuser with EPs. The following conclusions were drawn from current study:

- The results indicated that the adding energy promoters in planes 3,4 and 5 resulting in improve the S-shaped diffuser by increase the static pressure 
recovery and reduce the total pressure loss. Also, leading to delay the separation starting and eliminate the separation region.

- The highest performance enhancement when EPs installed at plane 5, the $C_{P R}$ has been increased by $28 \%$, the total loss coefficient $C_{T L}$ reduced by $18 \%$ compared to the bare S-shaped diffuser.

The authors acknowledge Universiti Teknologi PETRONAS (UTP) - Malaysia for the logistic and technical support to produce this work. The first author appreciates UTP financial support of his PhD study under TFA scheme. Appreciations are due to Ministry of Higher Education - Malaysia (MOHE) for permitting the use of the measuring instruments purchased under FRGS grant FRGS/1/2017/STG02/UTP/01/1.

\section{References}

1. Madadi, A., M. J. Kermani, and M. Nili-Ahmadabadi. Journal of Engineering for Gas Turbines and Power 136, no. 12, (2014)

2. Paul, A. R., Ranjan, P., Patel, V. K. and Jain, A. Aerospace Science and Technology 28, no. 1, 332-343. (2013)

3. Anderson, B. H., Huang, P. S., Paschal, W. A., and Cavatorta, E. Journal of Propulsion and Power, Vol. 8, No. 6, 1992, pp. 1266-1272. (1992).

4. Anderson, B. H., Huang, P.S., Paschal, W. A. and Cavatorta, E. Journal of Propulsion and Power 8, no. 6, 1266-1272. (1992)

5. Ashill, P., J. Fulker, and K. Hackett. In 39th Aerospace Sciences Meeting and Exhibit, p. 887. (2001)

6. Anabtawi, A., Blackwelder, R., Lissaman, P. and Liebeck, R. In $37^{\text {th }}$ Aerospace Sciences Meeting and Exhibit, p. 739. (1999)

7. Reichert, B. A, and Wendt B. J. In 31st Aerospace Sciences Meeting, p. 18. (1993)

8. Santner, C., GÃksttlich, E., Marn, A., Hubinka, J. and Paradiso, B. Journal of Turbomachinery 134, no. 1, 011023. (2012)

9. Zhang, Y., Hu, S., Zhang, X. F., Benner, M., Mahallati, A. and Vlasic, E. Journal of Engineering for Gas Turbines and Power 136, no. 11, 112604. (2014) 